\title{
Statistical Analysis of Goal Shooting of Football in 2012 European Cup Yan-xia $\mathrm{CAO}^{1, a}$ \\ Department of Physical Education, Yulin College , Yulin 719000, China \\ a916490618@qq.com
}

Keywords: The European Cup; Football; Goal Shooting; Statistical analysis

\begin{abstract}
The literature, logical analysis, video clips, and mathematical statistics methods are used in this paper for statistics and analysis 2012 European Cup Final stage 31 games 73 goals. The results showed that: Scoring high frequency period is the 60mins; the best area of goal is the goal area and penalty area. Means of attack mainly are break of flank and thrust down the middle. The goals of corner kick and free kick are used frequently in the game. Fast-paced goal, intense confrontation, rapid conversion of overall offensive and defensive are used frequently in the 2012 European Cup.

Four years of the European Cup is European football's top competition, even in the world of football also has a higher status. The top event of the statistical analysis, we can understand the status of football in Europe and better grasp the development trend in today's football. Due to modern football skills, the development of tactical play, the players individual combat capability and coordinated combat capability continues to improve, it is toward a comprehensive, flexible, fast, intense direction ${ }^{[1]}$. Ball on the offensive, not defensive ball, scoring goals and stop the opponent's offensive and defensive has become the focus of the game. Based on the 2012 European Cup 16 teams 31 games 73 goals (penalty kick showdown removed from the goal) of relevant indicators and statistics and analysis and comparative analysis of previous European Cup, European Cup to reveal the characteristics of scoring and explore its inherent laws, for the majority of coaches to guide the training and competition to provide a reference.
\end{abstract}

\section{Subjects and Methods}

\subsection{Subjects}

In the 2012 European Cup finals conducted in 31 games, a total of 73 goals (except for penalty goals) time Shi she area, shooting mode, initiating offensive manner and means as the object of study.

\subsection{Research Methods}

\subsubsection{Video statistics}

Summarize the current European Cup goals through 31 games feature live video and playback video observation and statistics, data analysis and reference portal.

\subsubsection{Literature}

Now the domestic and foreign related research papers football game Goal in terms of related works, this study provides a theoretical basis and methods.

\subsubsection{Comparative Analysis}

We will analyze the current situation of the European Cup goals in the first 12, 13 the European Cup goals contrast.

\subsubsection{Mathematical statistics}

We collected data related to the statistical processing using SPSS12.0 statistical software.

1.3 Scale Statistics

Field zoning: Goals video game based on observations and video clips, we will be divided into seven regions scored region. Zone 1 is the goal area; 2 area is restricted left side; Zone 3 of penalty area; 4 for the restricted area right rib; 5 Road area is outside the area; 6 area is outside the area on the left; 7 area is outside the area on the right. Goals zoning specifically shown in Figure 1. According to the game situation will attack the center and the stadium is divided into two wings, in 
the middle of the pitch 30m Road, both sides of the wing [2] (Fig. 2). Regions 1 and 3 further merger statistical analysis required for the closed road, Zone 2 and Zone 4 rib merged into the restricted area, District 6 and 7 are combined into restricted area outside the road, outside the area of District 5 Road, were recorded as I area, ii area, iv and iii region.

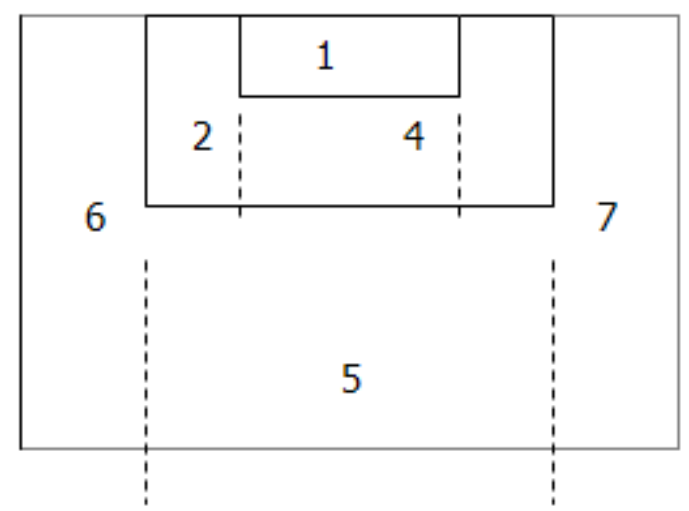

Figure 1 Goals zoning schematic diagram

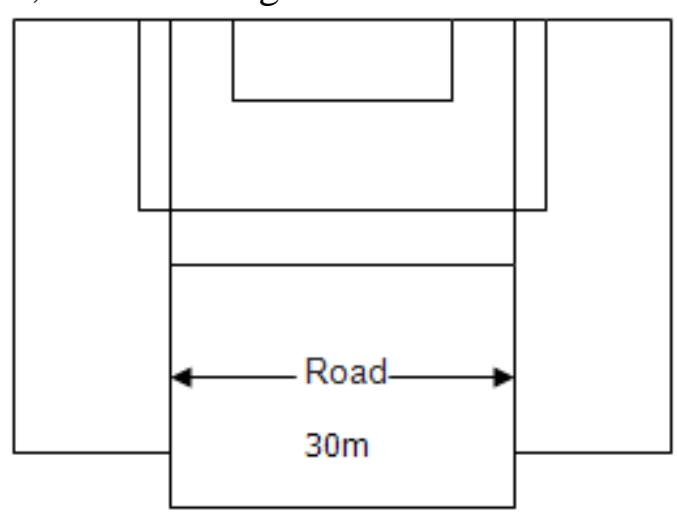

Figure 2 Offensive line schematic division

\section{Results and Analysis}

\subsection{The number shot on goal Statistical Analysis}

The current European Cup finals in 2012 June 9 to July 2, held in Poland and Ukraine. Carried out in all 31 games CCP fired 73 goals (not including the penalty kick showdown goal), an average of 2.35 goals per game, 16 teams completed a total of 880 shots, averaging 28.39 second shot, fired a total of 73 goals, scoring rate of $8.30 \%$, this figure is lower than the first three European Cup field goals (see Table 1). As can be seen, the modern football team pay more attention to defense in strengthening the effectiveness of the attack. Prevent other effective attack and shooting teams in tactical training and competition focused on training and use.

Table 12012 European Cup goals statistics and the number of shots

\begin{tabular}{ccccccc}
\hline \multirow{2}{*}{ Session times } & \multirow{2}{*}{ Event } & \multicolumn{3}{c}{ Shooting } & \multicolumn{3}{c}{ Goals } \\
\cline { 3 - 7 } & & $\mathrm{n}$ & $\bar{x}$ & $\mathrm{n}$ & $\bar{x}$ & $\%$ \\
\hline 11 Session ( 2000) & 31 & 815 & 26.29 & 85 & 2.74 & 10.43 \\
12 Session ( 2004) & 31 & 785 & 25.32 & 77 & 2.48 & 9.81 \\
13 Session ( 2008) & 31 & 892 & 28.77 & 77 & 2.48 & 8.62 \\
14 Session ( 2012 ) & 31 & 880 & 28.39 & 73 & 2.35 & 8.30 \\
\hline
\end{tabular}

2.2 Analysis of Scoring region

According to Table 2 and Table 3 shows that statistics[3], the current European Cup football Shishe area characterized mainly for $24.66 \%$ of the goals in the goal area (Zone 1) within Shishe completed, this proportion is higher than the first the proportion of the 17th World Cup goal in this area [4], zone 2, zone 4 respectively costal portion of the box, goals are low. Zone 3 is the goal area opposite the penalty area, scoring ratio in this area is $49.31 \%$, Zone 2 , Zone 3 , Zone 4 together constitute a restricted area, the proportion of the area of goal was $63.01 \%$ (including 3 penalty goals a), indicating that the area is the largest goal area, prompted the team scoring chances should be better grasp of the restricted area. District 5, District 6, District 7, mostly long-range area, less obvious goals. Modern football game against the fierce, scraping positive, or use long-range free kick in the attacking position when less than Jiugong goal directly, becoming a good way, but also fully explain football player on the football field of space and power of soccer game understanding. However, the current European Cup goals in IV area is 0, indicating that the area outside the road without a goal, a strong ball-handling skills of each team pass the ball and the ability to carry out long-range under the circumstances is not hasty, but by wing penetration, breakthrough, pass in the 
middle or seek shot at the goal area, such as the Spanish team often skilled combination passes to create goal opportunities.

Table 22012 European Cup regional statistics of Scoring

\begin{tabular}{|c|c|c|c|c|c|c|c|}
\hline & 1 Area & 2 Area & 3 Area & 4 Area & 5 Area & 6 Area & 7 Area \\
\hline $\mathrm{n}$ & 18 & 3 & 36 & 7 & 9 & 0 & 0 \\
\hline$\%$ & 24.66 & 4.11 & 49.31 & 9.59 & 12.33 & 0 & 0 \\
\hline \multicolumn{8}{|c|}{ Table 32012 European Cup Goal in the area of statistics } \\
\hline \multicolumn{2}{|c|}{ Session time } & I Area & II Area & & IIIArea & IVArea & Total \\
\hline 11 Session & $(2000)$ & 59 & 8 & & 11 & 7 & 85 \\
\hline 12 Session & (2004) & 56 & 6 & & 9 & 6 & 77 \\
\hline 13 Session & (2008) & 65 & 6 & & 6 & 0 & 77 \\
\hline 14 Session & (2012) & 54 & 10 & & 9 & 0 & 73 \\
\hline
\end{tabular}

2.3 Time Analysis of Scoring

According to FIFA Technical statistical methods, the goal time in the game, the second half, each $15 \mathrm{~min}$ as a time period, each game is divided into six time periods ago after statistics[5]. According to the actual situation, will fall into the first half stoppage time of the first $45 \mathrm{~min}$, into second half stoppage time of the first $90 \mathrm{~min}$, the second half stoppage time goal is also included in the appropriate time period extra time track the number of small, separate statistics[2]. Table 4 Statistics show that 73 goals in the current European Cup generated in $90 \mathrm{~min} 73$ ball game in hand, 0 goals in overtime, the first half goal total to 32 , the total number of goals $43.84 \%$, the total number of goals in the second half of $41,56.16 \%$ of the total number of goals. As can be seen the number of goals in the second half of the tournament is higher than the first half, with the first three games in the second half scored significantly higher than the number of features of the first half goals coincide. By Statistics found, near fourth European Cup goals in the second half of the average in the first 15 minutes to 15.25, indicating that each team after halftime through better physical recovery, and strive in the second half just It is harvested at the beginning, to strengthen the attack, increasing the number of shots.

Table 42012 European Cup Goal in the time table

\begin{tabular}{cccccccccc}
\hline Session time & $0-15$ & $16-30$ & $31-45$ & $46-60$ & $61-75$ & $76-90$ & $91-105$ & $106-120$ & Total \\
\hline 11 Session ( 2000 ) & 9 & 9 & 14 & 23 & 13 & 11 & 5 & 1 & 85 \\
12 Session ( 2004) & 6 & 15 & 9 & 12 & 12 & 16 & 5 & 2 & 77 \\
13 Session ( 2008) & 4 & 12 & 9 & 11 & 16 & 14 & 8 & 3 & 77 \\
14 Session ( 2012) & 7 & 13 & 12 & 15 & 13 & 13 & 0 & 0 & 73 \\
$\bar{x}$ & 6.5 & 10 & 11 & 15.25 & 13.5 & 13.5 & 4.5 & 1.5 & \\
\hline
\end{tabular}

2.4 Analysis of Scoring mode

The main technical means 73 European Cup goals this shot using statistics classified according to Table 5, the main use of technical means to score a direct shot, then control shot, head shot[6]. Wherein the goal scoring 25 goals, scored 27 goals then control shot, head shot scored 21 goals. Contrast 11, 12 and 13 th European Cup goals relevant information discovery, tournament players score, the more obvious decline in the proportion, while the proportion of the head to score improved significantly over the previous three ratio. Access control field goal ratio is higher than the proportion of the previous access control shot, but less than the 11th and 12th pick control shot ratio. Changes described above, each team defense more proactive, especially for finishing touches defense to foul tactics to undermine a potential threat even so Offence difficult to directly score. 
Table 52012 European Cup Adams shot scores Statistics

\begin{tabular}{|c|c|c|c|c|c|c|}
\hline \multirow{2}{*}{ Session time } & \multicolumn{2}{|c|}{ Direct shot } & \multicolumn{2}{|c|}{ Head shot } & \multicolumn{2}{|c|}{ Access control shot } \\
\hline & $\mathrm{n}$ & $\%$ & $\mathrm{n}$ & $\%$ & $\mathrm{n}$ & $\%$ \\
\hline 11 Session ( 2000 ) & 27 & 31.76 & 15 & 17.65 & 43 & 50.59 \\
\hline 12 Session ( 2004 ) & 26 & 33.77 & 17 & 22.08 & 34 & 44.16 \\
\hline 13 Session ( 2008 ) & 36 & 46.75 & 17 & 22.08 & 24 & 31.17 \\
\hline 14 Session ( 2012 ) & 25 & 34.25 & 21 & 28.77 & 27 & 36.98 \\
\hline
\end{tabular}

\subsection{Offensive Way Analysis}

Offensive way into a dynamic offensive attack and positioning the ball two kinds. Motion offense in accordance with the field area range is divided into the center and wing in two forms. Attacking is divided into: corner kick, free kick, penalty kick, throw four forms[2]. Table 6 Statistics show that the current World Cup wing attack on goal ratio of $43.42 \%$, the main wing attack to pass as the main way, players generally use the penalty area and scored from the wing edges between the range that is the implementation of Crossing better[7]; counter attack goal accounted for $28.95 \%$, in the middle attack mainly short pass penetration based, multi-player for the striker and the avant-garde of the tacit understanding. The number of visible goal motion offense under the form occupies a very high proportion, it shows that in today's dynamic world football offensive forms predominate. However, the current European Cup Soccer scores, the goal of positioning the ball 25 percent (Worthy of note is the current World Cup own goal also made two free kick caused) was significantly higher than the last game localization RALLY ratio. The above described changes in the modern football game, the players individual ability and the strength of the team to continuously improve the overall dynamic offensive attack is the preferred method for each team, but in the active defense defensive player even at the expense of foul against, the positioning ball is critical, the team attaches great importance to locate the ball, hoping by positioning the ball score. By positioning the ball scores become the current European Cup.

Table 6 Statistics of offensive way

\begin{tabular}{|c|c|c|c|c|c|c|c|c|c|}
\hline \multirow{2}{*}{ Way } & \multirow{2}{*}{ Mode } & \multicolumn{2}{|c|}{11 Session } & \multicolumn{2}{|c|}{12 Session } & \multicolumn{2}{|c|}{13 Session } & \multicolumn{2}{|c|}{14 Session } \\
\hline & & $\mathrm{n}$ & $\%$ & $\mathrm{n}$ & $\%$ & $\mathrm{n}$ & $\%$ & $\mathrm{n}$ & $\%$ \\
\hline \multirow{5}{*}{ Sidewalk } & Break through & 3 & 3.53 & 2 & 2.60 & 6 & 7.79 & 2 & 2.63 \\
\hline & penetration & 9 & 10.59 & 7 & 9.09 & 8 & 10.39 & 8 & 10.53 \\
\hline & Biography & 20 & 25.53 & 10 & 12.99 & 19 & 24.68 & 21 & 27.63 \\
\hline & Plagioclase pass & 5 & 5.88 & 2 & 2.60 & 1 & 1.30 & 2 & 2.63 \\
\hline & Total & 35 & 41.18 & 21 & 21.17 & 34 & 44.16 & 33 & 43.42 \\
\hline \multirow{4}{*}{ Road } & Break through & 8 & 9.41 & 11 & 14.29 & 7 & 9.90 & 2 & 2.63 \\
\hline & penetration & 10 & 11.76 & 11 & 14.29 & 10 & 12.99 & 18 & 23.68 \\
\hline & Long Passing & 1 & 1.18 & 7 & 9.09 & 13 & 16.88 & 2 & 2.63 \\
\hline & Total & 29 & 34.12 & 29 & 37.66 & 30 & 38.96 & 22 & 28.95 \\
\hline \multirow{5}{*}{$\begin{array}{c}\text { Positioning } \\
\text { ball }\end{array}$} & Corner kick & 3 & 3.53 & 12 & 15.58 & 5 & 6.94 & 8 & 10.53 \\
\hline & Free kick & 9 & 10.59 & 6 & 7.79 & 4 & 5.19 & 6 & 7.89 \\
\hline & Penalties & 8 & 9.41 & 7 & 9.09 & 4 & 5.19 & 3 & 3.95 \\
\hline & Throw & 0 & 0 & 0 & 0 & 0 & 0 & 2 & 2.63 \\
\hline & Total & 20 & 23.53 & 25 & 32.47 & 13 & 16.88 & 19 & 25 \\
\hline Own goals & & 1 & 1.18 & 2 & 2.60 & 0 & & 2 & 2.63 \\
\hline Total & & 85 & & 77 & & 77 & & 76 & \\
\hline
\end{tabular}


Note: Does not include the penalty kick showdown scoring, 14th in three penalty appeared at the group stage, and therefore be included in the statistical range

\section{Summary}

Modern football game, the goal area and goal area remains the main area of the front of the box score, score region to form the corner and the main short pass penetration, attack and defense of each team have been highly valued. Period goals from the point of view, on the beginning of the second half and the second half is coming to an end stage of a high-frequency period goals, the second half of the period corresponding to the number of goals is more than the number of goals in the first half, indicating that football game goal at a time having salient features. Continue to strengthen the role of defender, the overall offensive posture foregone conclusion. The last time before passing Shishe selection techniques used with a field goal, due to the ability to control the game players to strengthen, can directly pass directly transfer; direct shot directly on goal. If the success rate is not fast hit, the player handling the ball more rational development of the situation on the ground is determined more accurately. Diversity of attack at the same time pay more attention to the rationality and effectiveness of the offensive, the number of goals in the offensive movement on the rise, while the number of goals Attacking also an increasing trend, the overall dynamic offensive is still the team next major offensive way, pay more attention to positioning the ball attack success rate.

\section{Acknowlegments}

This work was financially supported "2015 Yulin Municipal Science and Technology Bureau Project(2015CYX_07)”

\section{Reference}

[1] Luo Biao, Dongchuan Guo. 2008 European Cup Goals Statistical Analysis [J]. World of Sports Academic Edition,2008,(11):23-24.

[2] Yu Yang, Lixian Qing, Li Qiuliang football game goal laws of [J]. Chengdu Institute of Physical Education, 2006 (3).

[3] Hu Yanfeng, Chen Fu Comparative Study of the 11th, 12th, 13th European Cup Football Championship Attack Situation [J]. EDUCATION GUIDE .2010,(1):125-126.

[4] Hou Weidong, Yang Dazhi. 17th World Cup Goal Statistics and Analysis [J]. Liaoning Sport Science and Technology, 2003 (2).

[5] Yuji Cheng, Xiao Jinyong. World Cup Goals spatial and temporal characteristics of [J]. Chengdu Institute of Physical Education, 2004 (4).

[6] He Xu. 17th World Cup Scoring Approaches [J]. Beijing Sports University, 2004 (1).

[7] Zhang Xiaochun, Liu Jianping 16th World Cup Goal analysis[J]. Wuhan Institute of Physical Education, 1999 (2). 